\title{
Mechanistic insights into cyclic peptide generation by DnaE split- inteins through quantitative and structural investigation
}

\author{
Leonhard M. Kick, Sabrina Harteis, Maximilian Koch and Sabine Schneider ${ }^{[\mathrm{a}]}$
}

\begin{abstract}
Inteins carry out protein-splicing reactions, which are utilised in protein chemistry, protein engineering and biotechnological applications. Rearrangement of the order of the domains in split-inteins results in a head-to-tail cyclisation of the target sequence, which can be used to genetically encode and express libraries of cyclic peptides. The efficiency of the splicing reaction depends on the target sequence. Here we used mass spectrometry to assess the cyclic peptide formation of different, hexameric target sequences in vivo by the DnaE split-inteins from Synechocystis sp. and Nostoc punctiforme, revealing a strong impact by the target sequence and intein on the intracellular peptide concentration. Furthermore, we determined the crystal structures of their pre-splicing complexes, which allowed us to identify F-Block Asp17 as crucial for the DnaE-mediated splicing reaction.
\end{abstract}

Inteins (internal protein), which are found in all domains of life, are single-turnover catalysts of posttranslational protein splice reactions. These intervening protein sequences self-excise themselves in a multistep autoprocessing event, followed by the ligation of the exteins (external protein) ${ }^{[1]}$ Moreover, the two intein halves and their respect exteins can also be encoded in different genomic locations, termed split-inteins. Here proteinsplicing and ligation is carried out in trans between the two separate precursors. ${ }^{[2]}$ In the last 20 years, methods for utilisation of inteins for protein engineering, chemical biology as well as pharmaceutical applications, such as expressed protein ligation and the semi-synthetic generation of modified proteins, have been developed. ${ }^{[3]}$ Furthermore, split-inteins can be used to genetically encode and express libraries of cyclic peptides (CPs), a method that was termed split-intein mediated circular ligation of peptides and proteins (SICLOPPS). ${ }^{[4]}$ Here the order of the two split-intein-domains is inverted and encoded as a single polypeptide, resulting in the head-to-tail cyclisation of the sequence between the IntC and IntN domains (Supporting Fig.1) Due to their cyclised backbone structure, CPs are extremely stable against proteolytic degradation and the entropic cost upon binding is reduced. ${ }^{[5]}$ Thus CPs provide useful scaffolds for biotechnological and pharmaceutical applications. ${ }^{[6]}$

Two naturally occurring, homologous split-inteins have been used for SICLOPPS in bacteria, yeast and human cells: the

[a] L. Kick ${ }^{+}$, S. Harteis ${ }^{+}$, M. Koch ${ }^{+}$, S. Schneider

Center for Integrated Protein Science

at the Department of Chemistry

Technische Universität München

Lichtenbergstrasse 4,

85748 Garching, Germany

E-mail: sabine.schneider@mytum.de

+ equal contribution

Supporting information for this article is given via a link at the end of the document.

DnaE split inteins from Nostoc punctiforme PCC73102 (Npu) and Synechocystis sp. PCC6803 (Ssp).[4b, 7] They share an overall sequence identity of $67 \%$ (N-domain) and $53 \%$ (Cdomain), respectively (Supporting Fig. 2). Despite the highly conserved molecular architecture and mechanism of action of inteins, they exhibit different splicing efficiencies and extein sequence preferences. The Ssp DnaE intein was shown to possess a relatively slow splicing velocity at the optimal reaction temperature of $30{ }^{\circ} \mathrm{C} .{ }^{[8]}$ In contrast, Npu DnaE has a 33-170 fold higher splicing rate and an overall yield ranging from $80-90 \% .{ }^{[9]}$ Its temperature optimum of $37^{\circ} \mathrm{C}$ is ideal for $\mathrm{CP}$ generation in Escherichia coli or human cell culture and was reported to be less influenced by the extein sequence adjacent to the essential Cys residue. ${ }^{[9 a, 9 c, 10]}$ Although engineered and other natural inteins were shown to possess higher splicing efficiency and to be partly less influenced by the extein sequences, ${ }^{[9 a, 11]}$ so far only the naturally occurring $\mathrm{Npu}$ and $\mathrm{Ssp}$ DnaE inteins have been used to generate expressed cyclic peptide libraries in screening campaigns. For such $\mathrm{CP}$ libraries, one needs to consider that the resulting intracellular $\mathrm{CP}$ concentration could impact on the outcome of in an in vivo screening assay.

To gain mechanistic insights into the in vivo CP generation by split-inteins, we quantitatively and structurally investigated the sequence bias of the Npu and Ssp DnaE inteins. By using synthetic ${ }^{13} \mathrm{C}$-labeled reference peptides and liquid chromatography mass spectrometry (LC/MS), we determined the amount of four different cyclic hexameric target sequences produced by the two homologous inteins in $E$. coli. Three of these sequences were chosen for their diversity in sequence (CFAHPQ, CGWIKA, CLNSLS), while the fourth peptide (CAFHPQ) was used to investigate the previously reported increased splicing efficiency with a Phe at the extein ${ }^{+2}$ position in Ssp DnaE. ${ }^{[12]}$ An equivalent Phe is also present in Npu, but it
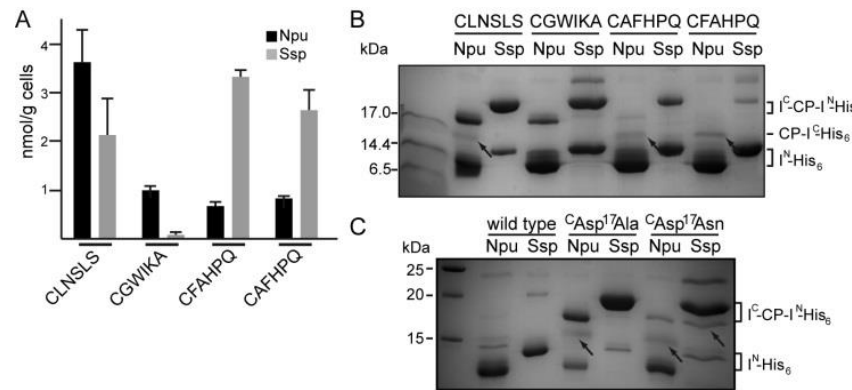

Figure 1. Hexameric cyclic peptide generation in E. coli and splicing efficiency of $\mathrm{Npu}$ und Ssp DnaE split-inteins. A) Quantification of four target CP sequences by LC-ESI-MS/MS in E. coli cell extracts. Measurements were repeated four times per extracts and average values with the respect standard deviations correspond to four biological replicates. B) Analysis of splicing efficiency of the purified DnaE inteins with four target sequences. C) Splicing efficiency of the purified DnaE-CFAHPQ wild type, ${ }^{C}$ Asp ${ }^{17} \mathrm{Ala}$ and ${ }^{\mathrm{C}} \mathrm{Asp}{ }^{17} \mathrm{Asn}$ mutant inteins. Mutation of the F-Block Asp to Ala strongly impedes DnaE splicing. The Npu DnaE ${ }^{\mathrm{D} 17 \mathrm{~N}}$ mutant shows slightly higher, residual activity. Ssp DnaE is more sensitive to ${ }^{\mathrm{C}} \mathrm{Asp}^{17}$-mutations. Highlighted by an arrow are the products of the premature cleavage at the IntC-splice junction. 
was shown that Npu DnaE splices even faster when the Phe is substituted with Trp or Met. ${ }^{[13]}$ Our quantification revealed that the overall yield of produced CP ranges from $0.1-3.7 \mathrm{nmol} / \mathrm{g}$ cells, depending on the used target sequence (Fig.1A and Supporting Table 1). In addition, the previously reported higher splicing efficiency of the Npu DnaE results in a higher yield of $\mathrm{CP}$ for two of the four hexameric target sequences. However, we used here the Ssp DnaE ${ }^{\mathrm{A} 35 \mathrm{H}}$-intein variant shown to enhance the splicing velocity as published for the SICLOPPS method. ${ }^{[4 a]}$

The amounts of produced CP can be correlated with the ratios between spliced and pre-spliced proteins for the Ssp DnaE intein: While almost complete processing can be seen for the CFAHPQ-target sequence, only about one third of the protein has undergone splicing with the CAFHPQ peptide, albeit the yields for the CFAHPQ and CAFHPQ sequences are similar with 3.3 and $2.7 \mathrm{nmol} / \mathrm{g}$ cells (Fig. $1 \mathrm{~A}$ and Supporting Table 1). The CFAHPQ and CAFHPQ target peptide for both inteins is encoded by the same DNA sequence without any rare codons (Supporting Table 2). For the Npu DnaE intein apparently no direct link between the splicing ratios and produced CPs can be observed. However, analysis by immunoblotting revealed that Npu DnaE-CLNSLS might be marginally higher expressed than the CAFHPQ and CFAHPQ constructs (Fig. 1B and Supporting Fig. 3). In addition, premature cleavage at the C-terminal splice junction can be seen for the three Npu DnaE target sequence constructs with the lowest peptide yields (CGWIKA, CFAHPQ, CAFHPQ), but not for the CLNSLS peptide or in the Ssp DnaE intein (Fig. 1B). Early, off-pathway C-terminal cleavage, which is caused by the cyclisation of the inteins C-terminal Asn prior to branch intermediate formation, arises when coordination of the reaction steps is either perturbed by mutation or by expression between foreign exteins. ${ }^{[14]}$ Furthermore, it was recently shown that Npu DnaE expression can be toxic for E. coli cells. ${ }^{[5 b]}$ Taken together the overall intracellular concentration of the CP product depends on the expression level, premature cleavage at the $\mathrm{C}$ terminal splice junction, possible toxic effects and the sequencedependent splicing efficiencies. Moreover, the pre-organisation imposed by the short hexapeptide target sequence, due to steric restraints, possibly influences the splicing reaction. With short target peptides the impact of the extein sequence is likely to be higher compared to longer, more flexible target sequences. ${ }^{\text {[9b, 15] }}$

To gain insights into the molecular mechanism of the splicing reactions, we determined the crystal structures of the catalytically incompetent pre-splice complexes of both splitinteins $\mathrm{Npu}$ DnaE ${ }^{\mathrm{C} 1 \mathrm{~A} / \mathrm{N} 36 \mathrm{~A}}$ and $\mathrm{S} s p \mathrm{DnaE}^{\mathrm{C} 1 \mathrm{~A} / \mathrm{A} 35 \mathrm{H} / \mathrm{N} 36 \mathrm{~A}}$, with the target sequences CAFHPQ and CFAHPQ. To our best knowledge, these are the first fully resolved structures of inteinextein complexes, showing the organisation of the splice junctions prior to the splicing reaction. All DnaE inteins investigated here adopt the typical horse-shoe like folding topology, with the hexameric target sequence protruding from the catalytic side (Fig. 2A and Supporting Fig. 4). Furthermore, a second conformation is observed in the crystal of Ssp DnaE ${ }^{\mathrm{C} 1 \mathrm{~A} / \mathrm{A} 35 \mathrm{H} / \mathrm{N} 36 \mathrm{~A}}$-CAFHPQ (Fig. 2B) trapped by an intermolecular disulphide-bridge between the $\mathrm{Cys}^{+1}$ of two proteins at the C-terminal splice junction (Supporting Fig. 4). For Npu DnaE a "capture and collapse"-intein-folding mechanism was postulated, were the second IntN-lobe attacks the first IntC-part, forming a native-like intermediate, which is followed by a collapse of this intermediate onto the first IntN-lobe. ${ }^{[16]}$ In our trapped structure the IntC-IntN subdomain needs to rotate 89 around the bending residues $52-54\left(\operatorname{IntN} ;{ }^{N} \beta 5\right)$ and 10-13 (IntC, ${ }^{c} \beta 1$ ), in order to form the fully assembled intein (Fig. 2B). This could have resemblance to the predicted native-like folding intermediate of intein-folding model-mechanism "capture and collapse", ${ }^{16]}$ albeit we cannot proof that the here observed conformation is directly on the folding pathway.

In all here reported structures, the hexameric target peptides are engaged in intermolecular crystal contacts (Supporting Fig. 6-8), which likely impact on torsion angles. However, our structures of the Npu and Ssp DnaE-pre-splice complexes provide further insights into the interconnected and complex hydrogen bonding network at the splice junctions that is responsible for the DnaEmediated protein-splicing reaction. ${ }^{[3 \mathrm{~d}]}$ The orientation of ${ }^{\mathrm{C}} \mathrm{Ala}^{36}$ and ${ }^{\mathrm{N}} \mathrm{Ala}^{1}$ (C $\beta$-atoms), which correspond to ${ }^{\mathrm{C}} \mathrm{Asn}^{36}$ and ${ }^{\mathrm{N}} \mathrm{C}^{1}$ in the active inteins, are equivalent orientated as found in other available wild type intein structures. Thus, we modelled the sidechain atoms of ${ }^{\mathrm{C}} \mathrm{Asn}{ }^{36}$ and ${ }^{\mathrm{N}} \mathrm{C} 1$ stereo chemically into our mutant intein structures (Supporting Fig. 9+10). The distances between the $\mathrm{S}_{\gamma}\left({ }^{\mathrm{N}} \mathrm{C} 1\right)$ and the $\mathrm{C}$-terminal splice junction range from $\sim 8-10 \AA$. Such distances observed in the structures of other inteins were argued to correspond to an "open conformation" of the splice junctions ${ }^{[17]}$ (Supporting Fig. 11+12, Supporting Table 3).

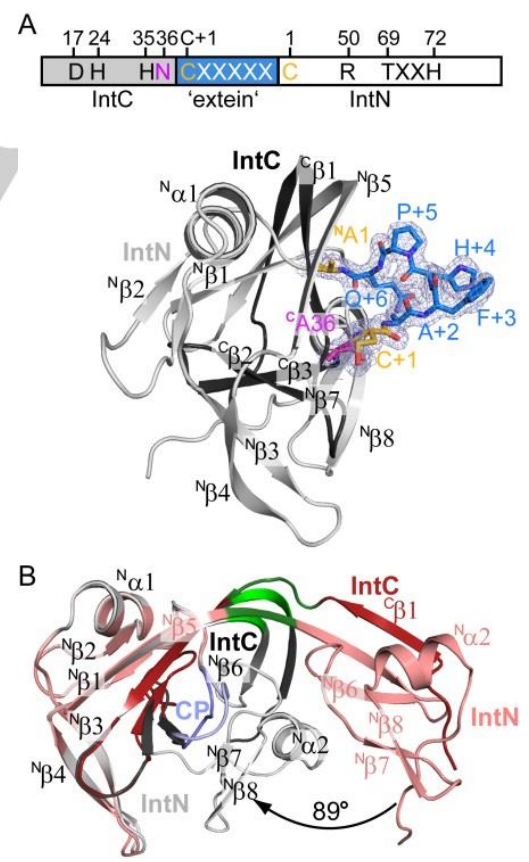

Figure 2 Structure of DnaE-pre-splicing complex. A) Schematic representation of the intein pre-splicing complex, in which important sequence motifs are highlighted. Below: X-ray crystal structure of the $S s p$ DnaE ${ }^{\mathrm{C} 1 \mathrm{~A} / \mathrm{A} 35 \mathrm{H} / \mathrm{N} 36 \mathrm{~A}}$. CAFHPQ pre-splicing complex. The split-intein is dawn as ribbon (IntN: grey; IntC: black), the hexameric target cyclic peptid sequence (blue), ${ }^{\mathrm{C}} \mathrm{Ala}^{36}$ (pink), $\mathrm{C}^{+1}$ and ${ }^{N} \mathrm{Ala}^{1}$ (golden) are highlighted as sticks. The $\mathrm{F}_{\mathrm{o}}-D \mathrm{~F}_{\mathrm{c}}$ simulated annealing difference electron density map, contoured at $2.5 \sigma$, is depicted as blue mesh. B) The two conformation observed in the crystal structures of the Ssp DnaE ${ }^{\mathrm{C} 1 \mathrm{~A} / \mathrm{A} 35 \mathrm{H} / \mathrm{N} 36 \mathrm{~A}}$-CFAHPQ pre-splicing complex, representing the fully assembled intein (grey) and a second conformation(red), possibly resembling the intermediate of the "capture and collapse"-mechanism of intein folding. Highlighted in green are the bending residues (IntN 52-54, IntC 10-13). Please note: crystallisation experiments were set up in the presence of reducing agent, with monomeric and monodispersed protein (Supporting Fig. 5). 


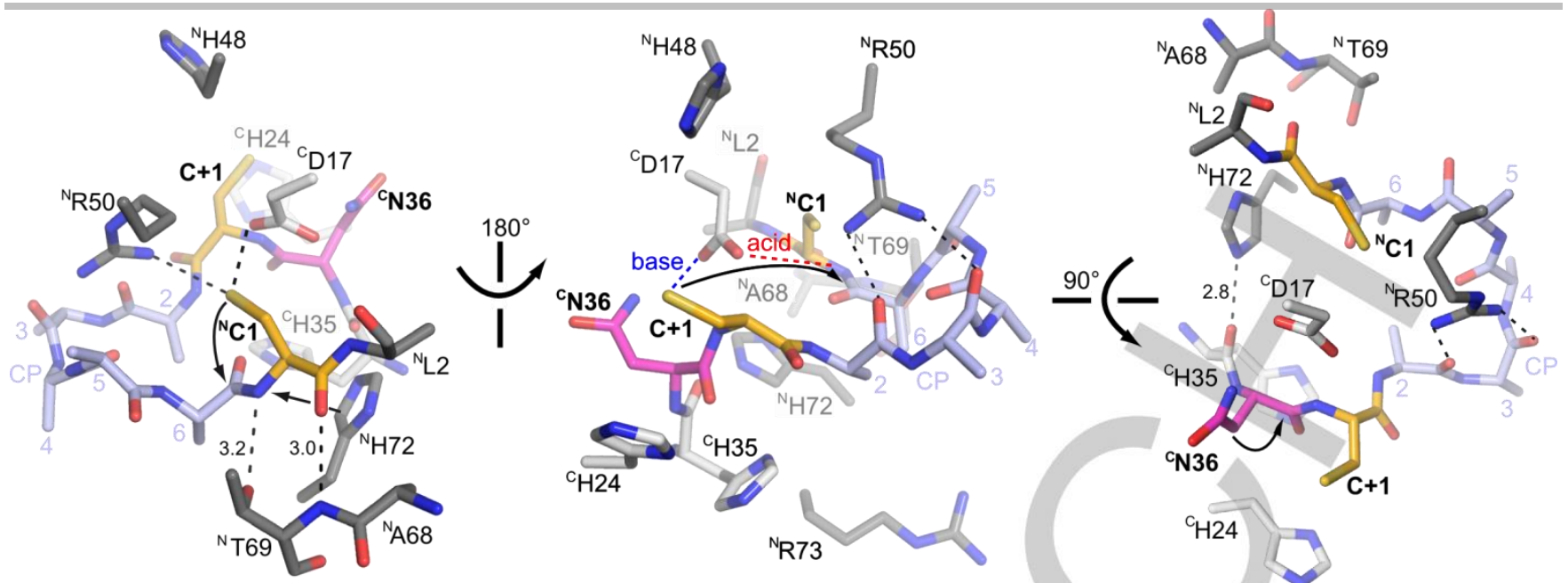

Figure 3 Role of amino acid residues in the different steps of the protein splicing reaction based on the X-ray crystal structure of the Ssp DnaE ${ }^{\mathrm{C} 1 \mathrm{~A} / \mathrm{A} 35 \mathrm{H} / \mathrm{N} 36 \mathrm{~A}}$-presplicing complex. Residues involved in S-N-acyl shift (left), transthioesterfication (middle) and Asn-cyclisation (right) are highlighted. The carbon atoms of the cyclic target sequence are coloured in light blue, the IntN domain in dark grey, the IntC domain light grey, ${ }^{\mathrm{C}} \mathrm{Asn}^{36}$ in pink, ${ }^{\mathrm{N}} \mathrm{C}^{1}$ and $\mathrm{Cys}{ }^{+1}$ in golden. Please note that the side chain atoms for ${ }^{\mathrm{C}} \mathrm{Asn}^{36}$ and ${ }^{\mathrm{N}} \mathrm{C}^{1}$ are not present in our expression construct, and thus were modelled stereo chemically, based on the comparison with other available intein structures. For clarity, we have removed the oxygen from the Cys ${ }^{+1}$, which is oxidised in our structure.

The first step of the protein-splice reaction is the energetically unfavoured N-S-acyl shift at the N-terminal splice junction. The nucleophile $\left({ }^{\mathrm{N}} \mathrm{Cys}{ }^{1}\right)$ needs to be deprotonated by a base and the ground state is destabilised. ${ }^{[3 \mathrm{~d}]}$ Here, a direct interaction of the peptide bond at the splice-junction with the amid-nitrogen and $\mathrm{O}^{\gamma}$ of ${ }^{\mathrm{N}} \mathrm{Thr}^{69}$ mediates the ground state destabilisation and positions the splice junction (Fig. 3, left). It was previously shown for DnaE, that ${ }^{\mathrm{N}} \mathrm{Thr}{ }^{69}$ likely enhances the rate of the N-S acyl shift through this interaction. ${ }^{[18]}$

In the Mycobacterium tuberculosis (Mtu) RecA intein $\mathrm{His}^{73}$ and Asp ${ }^{422}$ (in DnaE corresponding to ${ }^{\mathrm{N}} \mathrm{His}^{72}$ and ${ }^{\mathrm{C}} \mathrm{Asp}^{17}$; Supporting Fig. 11+12) undergo large $p K a$ shifts during protein splicing and are essential for the required acid-base catalysis. ${ }^{[19]}$ Mutation of ${ }^{\mathrm{C}}$ Asp ${ }^{17}$ to Ala impedes splicing in both inteins, whereas the substitution of ${ }^{\mathrm{C}} \mathrm{Asp}{ }^{17}$ by Asn retained some splicing activity in Npu DnaE, but not in Ssp DnaE (Fig. 1C). While for Ssp DnaE such a pKa shift appears to be necessary, for $\mathrm{Npu}$ DnaE replacement of the charged Asp with a polar Asn is sufficient to maintain some protein splicing activity during the $16 \mathrm{~h}$ expression time. The Asn could here fulfil a structural or chargestabilising role. In addition, for both $\mathrm{Npu}$ DnaE mutants the same extend of premature cleavage at the C-terminal junction can be observed. Asn cyclisation resulting in premature C-terminal cleavage was previously reported for the otherwise splicing deficient Npu DnaE ${ }^{\mathrm{N}} \mathrm{Cys}^{1}$ to Ala mutant. ${ }^{[9 b]}$ In contrast, Cterminal cleavage in the Ssp DnaE only occurs in the ${ }^{\mathrm{C}} \mathrm{Asp}^{17}$ to Asn, but not the ${ }^{\mathrm{C}} \mathrm{Asp}^{17}$ to Ala mutant (Fig. 1C). Furthermore, it was shown for both inteins that mutation of residues outside of the active side can positively and negatively modulate the splicing efficiency, most likely due to the perturbation of amino acid networks. ${ }^{[11,20]}$

Our data show ${ }^{\mathrm{C}} \mathrm{Asp}^{17}$ is important for DnaE-mediated protein splicing in CP generation. In our structures, ${ }^{\mathrm{N}} \mathrm{His}^{72}$ is located below the splice junction, too far away from the ${ }^{\mathrm{N}} \mathrm{Cys}{ }^{1}$-side chain for its deprotonation. Nevertheless, deprotonation could be carried out by the action of ${ }^{\mathrm{C}} \mathrm{Asp}^{17}$. ${ }^{\mathrm{N}} \mathrm{His}^{72}$ would then be ideally positioned to support the destabilisation of the ground state ${ }^{[21]}$ and the protonation of the tetrahedral intermediate. In addition, ${ }^{\mathrm{C}} \mathrm{Asp}^{17}$ could also activate $\mathrm{Cys}^{+1}$ for the following transthioesterfication step by deprotonation, and act as an acid to mediate bond-cleavage, resulting in the branched (or lariat) intermediate (Fig. 3, middle). Such a role was postulated for $\mathrm{Asp}^{422}$ in Mtu RecA. ${ }^{[19 b, 22]}$ In the Ssp DnaB mini-intein the deprotonation of Asn is mediated by an Asp, followed by an attack on the C-scissile peptide bond and subsequen cyclisation. ${ }^{[23]}$ Our structure reveals that ${ }^{\mathrm{C}} \mathrm{Asp}^{17}$ is indeed positioned, that is could deprotonate ${ }^{\mathrm{C}} \mathrm{Asn}^{36}$ (Fig. 3, right). Ground state destabilisation of the C-terminal splice junction for the Asn cyclisation possibly occurs through the conserved ${ }^{\mathrm{C}} \mathrm{His}^{24}$ and the action of the penultimate ${ }^{\mathrm{C}} \mathrm{His} / \mathrm{Ser}^{35}$, shown to be important for efficient splicing of DnaE split-inteins. ${ }^{[24]}$ Furthermore, ${ }^{\mathrm{C}} \mathrm{His} / \mathrm{Ser}^{35}$ are likely not only to stabilise the tetrahedral intermediate, but also to protonate the amide nitrogen of the scissile peptide bond, turning it a good leaving group. ${ }^{[25]}$ An Ala at this position, analogue to the native Ssp DnaE intein, is not able to fulfil this function.

In addition, ${ }^{\mathrm{N}} \mathrm{Arg}^{50}$ was shown to act positively on splicing. ${ }^{[26}$ According to our structure ${ }^{\mathrm{N}} \mathrm{Arg}^{50}$ could influence the orientation of ${ }^{\mathrm{N}} \mathrm{Cys}^{1},{ }^{\mathrm{C}} \mathrm{Asp}^{17}$ and/or the exteins peptide backbone (Fig. 3 and Supporting Fig. 6). The last step in protein splicing is the uncatalysed $\mathrm{S}-\mathrm{N}$-acyl shift to rearrange to the more stable amide bond, which does not need support by surrounding residues.

In summary, our results show that intracellular concentrations of genetically encoded CP produced by Ssp and $\mathrm{Npu}$ DnaE inteins largely vary, depending on the extein sequence. Nevertheless, both inteins were successfully used to generate $\mathrm{CP}$ libraries in screening campaigns. Furthermore, we provide mechanistic insight into the expressed CP generation by these two inteins, identifying the F-Block ${ }^{\mathrm{C}} \mathrm{Asp} 17$ is crucial for efficient splicing of linear peptides in $\mathrm{Npu}$ and Ssp DnaE intein.

\section{Experimental Section}

Chemical synthesis of unlabelled and ${ }^{13} \mathrm{C}$-labeled reference cyclic peptides was done by Fmoc-solid phase peptide synthesis, followed by cyclisation in solution. ${ }^{[27]}$ The DnaE split-Inteins from Nostoc punctiforme PCC73102 and Synechocystis sp. PCC6803 were expressed in Escherichia coli. Cyclic peptides were extracted using butanol, the cell extracts spiked with the respect ${ }^{13} \mathrm{C}$-labeled reference peptides and 
analysed by HPLC-ESI-MS/MS (Supporting Fig. 13-18). For crystallisation catalytically inactive variants $S s p$ DnaE ${ }^{\mathrm{C} 1 \mathrm{~A} / \mathrm{A} 35 \mathrm{H} / \mathrm{N} 36 \mathrm{~A}}$ and Npu DnaE ${ }^{\mathrm{C} 1 \mathrm{~A} / \mathrm{N} 36 \mathrm{~A}}$ were expressed, purified and crystallised. Structures were solved by molecular replacement. The structure factors and models have been deposited in the PDB (accession codes: 5OL1, 5OL5, 5OL6, 5OL7) For detailed materials and methods see the Supporting Information online.

\section{Acknowledgements}

This work was supported by the Fonds der chemischen Industrie, the Deutsche Forschungsgemeinschaft (SCHN 1273, SFB749) and the Center for Integrated Protein Science Munich (CIPSM). We thank S. A. Sieber and M. Groll for access to the mass spectrometry and crystallisation facilities, respectively. We also thank the Swiss Light Source (SLS), European Synchrotron Radiation Facility (ESRF) and the Deutsches Elektronen Synchrotron (DESY) PETRA III for beamtime and the staff of beamlines PXI (SLS), ID23-2, ID30B, MASSIF-1 (ESRF) and P14 (PETRA III) for assistance with the crystal testing and data collection. We thank Gregor Witte for carrying out data collection at P14.

Keywords: split-intein $\bullet$ protein splicing $\bullet$ cyclic peptide $\bullet$ intein structure

[1] F. B. Perler, E. O. Davis, G. E. Dean, F. S. Gimble, W. E. Jack, N. Neff, C. J. Noren, J. Thorner, M. Belfort, Nucleic Acids Res. 1994, 22, 1125 1127

[2] A. S. Aranko, A. Wlodawer, H. Iwai, Protein Eng. Des. Sel. 2014, 27, 263-271.

[3] a) M. Cheriyan, F. B. Perler, Adv Drug Deliv Rev 2009, 61, 899-907; b) S. Elleuche, S. Poggeler, Appl. Microbiol. Biotechnol. 2010, 87, 479 489; c) M. Vila-Perello, T. W. Muir, Cell 2010, 143, 191-200; d) G. Volkmann, H. D. Mootz, Cell Mol. Life Sci. 2013, 70, 1185-1206; e) N. H. Shah, T. W. Muir, Chem Sci. 2014, 5, 446-461.

[4] a) C. P. Scott, E. Abel-Santos, M. Wall, D. C. Wahnon, S. J. Benkovic, Proc. Natl. Acad. Sci. U. S. A. 1999, 96, 13638-13643; b) A. Tavassoli, S. J. Benkovic, Nat. Protoc. 2007, 2, 1126-1133.

[5] a) D. J. Craik, Science 2006, 311, 1563-1564; b) J. E. Townend, A Tavassoli, ACS Chem. Biol. 2016, 11, 1624-1630.

[6] a) T. Kawakami, A. Ohta, M. Ohuchi, H. Ashigai, H. Murakami, H. Suga, Nat Chem Biol 2009, 5, 888-890; b) A. Tavassoli, Curr. Opin. Chem. Biol. 2017, 38, 30-35; c) A. Zorzi, K. Deyle, C. Heinis, Curr. Opin. Chem. Biol. 2017, 38, 24-29.

[7] a) T. M. Kinsella, C. T. Ohashi, A. G. Harder, G. C. Yam, W. Li, B. Peelle, E. S. Pali, M. K. Bennett, S. M. Molineaux, D. A. Anderson, et al., J. Biol. Chem. 2002, 277, 37512-37518; b) J. A. Kritzer, S Hamamichi, J. M. McCaffery, S. Santagata, T. A. Naumann, K. A Caldwell, G. A. Caldwell, S. Lindquist, Nat. Chem. Biol. 2009, 5, 655663 ; c) K. Jagadish, A. Gould, R. Borra, S. Majumder, Z. Mushtaq, A. Shekhtman, J. A. Camarero, Angew. Chem. Int. Ed. Engl. 2015.

[8] a) D. D. Martin, M. Q. Xu, T. C. Evans, Jr., Biochemistry 2001, 40, 1393-1402; b) N. M. Nichols, J. S. Benner, D. D. Martin, T. C. Evans, Jr., Biochemistry 2003, 42, 5301-5311.

[9] a) H. Iwai, S. Zuger, J. Jin, P. H. Tam, FEBS letters 2006, 580, 1853 1858; b) N. H. Shah, E. Eryilmaz, D. Cowburn, T. W. Muir, J. Am. Chem. Soc. 2013, 135, 5839-5847; c) J. Zettler, V. Schutz, H. D. Mootz, FEBS letters 2009, 583, 909-914.

[10] P. Carvajal-Vallejos, R. Pallisse, H. D. Mootz, S. R. Schmidt, The J. Biol. Chem. 2012, 287, 28686-28696.

[11] S. W. Lockless, T. W. Muir, Proc. Natl. Acad. Sci. U. S. A. 2009, 106, 10999-11004.

[12] T. A. Naumann, S. N. Savinov, S. J. Benkovic, Biotechnol. Bioeng 2005, 92, 820-830

[13] M. Cheriyan, C. S. Pedamallu, K. Tori, F. Perler, J. Biol. Chem. 2013 288, 6202-6211.

[14] K. V. Mills, M. A. Johnson, F. B. Perler, J. Biol. Chem. 2014, 289 14498-14505.

[15] K. Jagadish, R. Borra, V. Lacey, S. Majumder, A. Shekhtman, L. Wang J. A. Camarero, Angew. Chem. Int. Ed. Engl. 2013, 52, 3126-3131.

[16] N. H. Shah, E. Eryilmaz, D. Cowburn, T. W. Muir, J. Am. Chem. Soc 2013, 135, 18673-18681.

[17] J. S. Oeemig, D. Zhou, T. Kajander, A. Wlodawer, H. Iwai, J. Mol. Biol 2012, 421, 85-99.

[18] A. K. Dearden, B. Callahan, P. V. Roey, Z. Li, U. Kumar, M. Belfort, S K. Nayak, Protein Sci. 2013, 22, 557-563.

[19] a) Z. Du, P. T. Shemella, Y. Liu, S. A. McCallum, B. Pereira, S. K Nayak, G. Belfort, M. Belfort, C. Wang, J Am Chem Soc 2009, 131 11581-11589; b) Z. Du, Y. Zheng, M. Patterson, Y. Liu, C. Wang, J Am Chem Soc 2011, 133, 10275-10282.

[20] a) D. W. Wood, W. Wu, G. Belfort, V. Derbyshire, M. Belfort, Nat Biotechnol. 1999, 17, 889-892; b) E. Adam, F. B. Perler, J. Mol. Microb. Biotech. 2002, 4, 479-487.

[21] A. Romanelli, A. Shekhtman, D. Cowburn, T. W. Muir, Proceedings of the National Academy of Sciences of the United States of America 2004, 101, 6397-6402.

[22] B. Pereira, P. T. Shemella, G. Amitai, G. Belfort, S. K. Nayak, M. Belfort $J \mathrm{Mol}$ Biol 2011, 406, 430-442.

[23] J. I. Mujika, X. Lopez, A. J. Mulholland, J. Phys. Chem. B 2009, 113 5607-5616.

[24] N. H. Shah, G. P. Dann, M. Vila-Perello, Z. Liu, T. W. Muir, J. Am. Chem. Soc. 2012, 134, 11338-11341.

[25] a) S. Mathys, T. C. Evans, I. C. Chute, H. Wu S. Chong, J. Benner, X. Q. Liu, M. Q. Xu, Gene 1999, 231, 1-13; b) D. W. Wood, V. Derbyshire, W. Wu, M. Chartrain, M. Belfort, G. Belfort, Biotechnol. Prog. 2000, 16 1055-1063.

[26] Q. Wu, Z. Gao, Y. Wei, G. Ma, Y. Zheng, Y. Dong, Y. Liu, Biochem. J. 2014, 461, 247-255.

[27] S. Zimmer, E. Hoffmann, G. Jung, H. Kessler, Liebigs Ann. Chem. 1993 497-501. 


\section{Entry for the Table of Contents}

\section{COMMUNICATION}

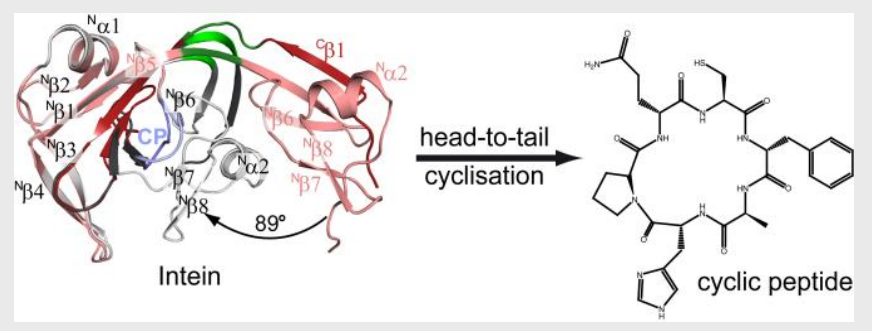

Leonhard Kick, Sabrina Harteis,

Maximilian Koch and Sabine Schneider ${ }^{*}$

Page No. - Page No.

Inteins carry out protein-splicing reactions and can be used to genetically encode and express cyclic peptides. We show that the target sequence and DnaE-intein strongly impact on the intracellular peptide concentration. The crystal structures of the intein pre-splicing complexes provide further molecular details on DnaE-mediated protein splicing. 